This item was submitted to Loughborough's Research Repository by the author.

Items in Figshare are protected by copyright, with all rights reserved, unless otherwise indicated.

\title{
Exploring inattention and distraction in the SafetyNet accident causation
} database

PLEASE CITE THE PUBLISHED VERSION

PUBLISHER

SAFER and INRETS

VERSION

NA (Not Applicable or Unknown)

LICENCE

CC BY-NC-ND 4.0

\section{REPOSITORY RECORD}

Talbot, Rachel, and Helen Fagerlind. 2019. "Exploring Inattention and Distraction in the Safetynet Accident Causation Database". figshare. https://hdl.handle.net/2134/6265. 
This item was submitted to Loughborough's Institutional Repository (https://dspace.lboro.ac.uk/) by the author and is made available under the following Creative Commons Licence conditions.

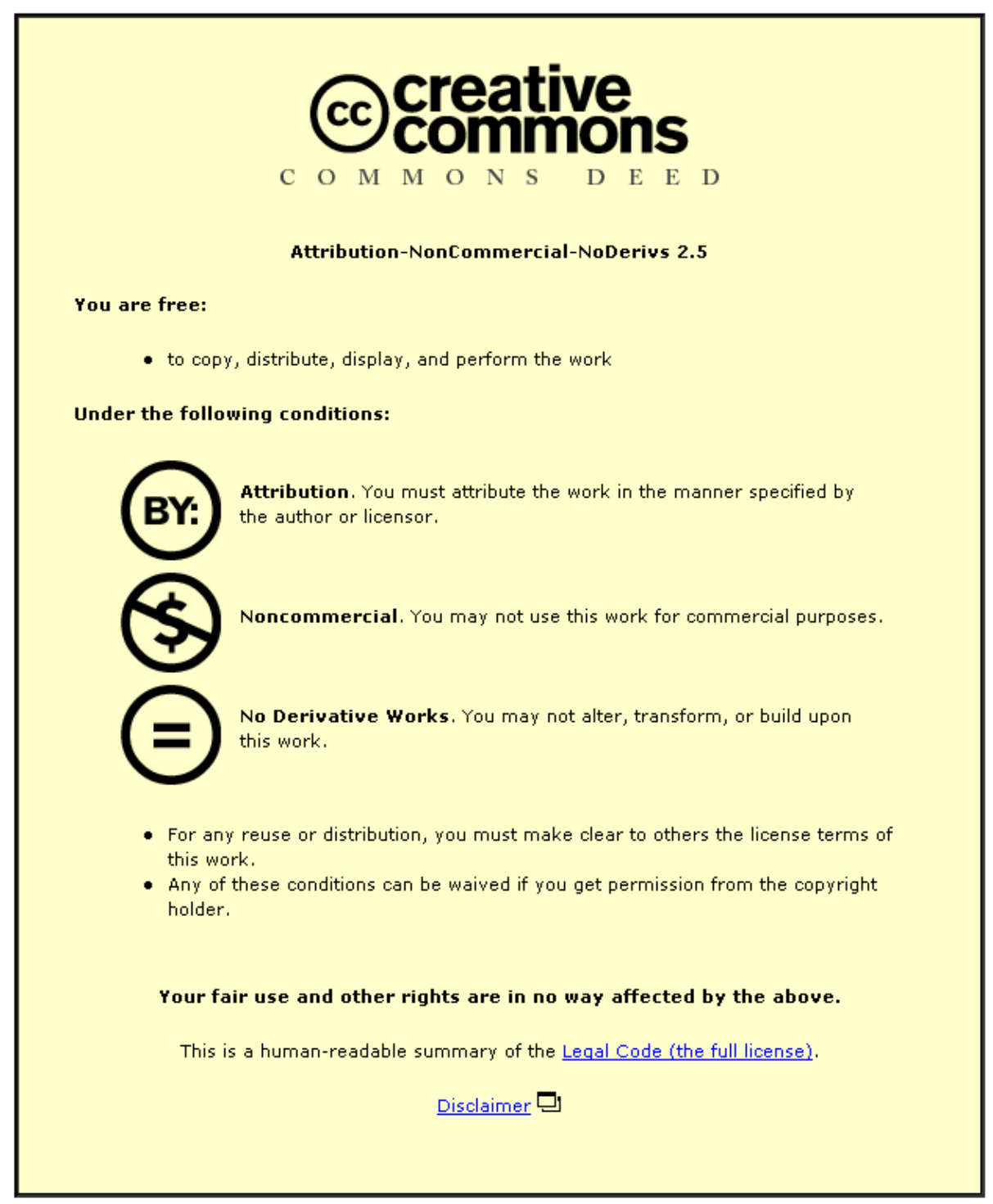

For the full text of this licence, please go to: http://creativecommons.org/licenses/by-nc-nd/2.5/ 


\title{
EXPLORING INATTENTION AND DISTRACTION IN THE SAFETYNET ACCIDENT CAUSATION DATABASE
}

\author{
R Talbot ${ }^{1^{*}}$, H Fagerlind $^{2}$ \\ 1. Vehicle Safety Research Centre, Loughborough University, Ashby Road, Loughborough, \\ LE11 3TU, UK. Tel. +44 (0)1509 226954, Email. r.k.talbot@lboro.ac.uk \\ 2. Chalmers University of Technology, Gothenburg, Sweden
}

\begin{abstract}
The SafetyNet Accident Causation Database was formulated as part of the SafetyNet Integrated Project. Data were collected in 6 European countries using 'on-scene' and 'nearly on-scene' crash investigation methods. $32 \%$ of crashes recorded in the Database, involved at least one driver, rider or pedestrian, which had been assigned the SNACS codes 'Inattention' and/or 'Distraction'. 212 of the drivers were assigned 'Distraction' and 140 drivers were given the code 'Inattention'. Distraction and Inattention often leads to missed observations and 'Timing' or 'Direction' critical events. In addition, the type of distraction and inattention differs according to the crash type.
\end{abstract}

\section{KEYWORDS}

Accident Causation, Distraction, Inattention

\section{INTRODUCTION}

The SafetyNet Accident Causation Database was formulated as part of the large European Commission supported $6^{\text {th }}$ framework project SafetyNet. The aim of SafetyNet was to build a framework for the European Road Safety Observatory as well as to collect new data and to develop new data collection methodologies. SafetyNet comprised of seven work packages that covered three areas of work namely, 'Macroscopic Data', 'In-depth Data' and 'Data Application'. The SafetyNet Accident Causation Database is one of two databases developed as part of the 'Indepth' data area. The second database was the SafetyNet Fatal Accident Database.

The SafetyNet Accident Causation Database was populated with data collected in six European countries using on-scene or nearly on-scene methodologies. Causation data was recorded according to the SafetyNet Accident Causation System (SNACS), a method that was also developed as part of the SafetyNet project. Distraction and inattention are both included as distinct contributory factors within the SNACS methodology.

SNACS analyses are performed on a vehicle level and SNACS charts are assigned according to the contributory factors that can be attributed to the driver, rider or 
pedestrian. These charts were used to identify drivers, riders and pedestrians in the database that had been assigned the SNACS code 'Distraction' or 'Inattention'.

The following sections will describe the SafetyNet Accident Causation Database and explain the SNACS method in more detail. 'Distraction' and 'Inattention' will then be explored by examining the contributory factor patterns for drivers/riders/pedestrians that were assigned 'Distraction' and/or 'Inattention' and looking at the vehicle trajectories associated with these drivers/riders/pedestrians. 'Distraction' and 'Inattention' will be explored further by looking at the sub-categories of these SNACS codes that were assigned to the drivers/riders/pedestrians.

\section{METHODS}

\section{SafetyNet Accident Causation Database}

The final version of the SafetyNet Accident Causation Database contains 1005 cases, 1828 vehicles and 2422 road users. The crash data stored in the database were collected by six crash investigation teams operating in Germany, Italy, The Netherlands, Finland, Sweden and the UK. All types of crash and road user were eligible to be included in the database. Crashes were collected during the investigation teams' operational periods not all of which operated at night. Data was collected using 'on-scene' or 'nearly on-scene' methodologies. This means that investigation teams attended the scene of the crash either while the vehicles were still in their post-crash rest positions or within a few hours or days of the crash. Multidisciplinary teams conducted examinations of the road environment and the involved vehicles. Teams also aimed to talk to all involved road users.

The Accident Causation Database includes both general variables (aspects of the crash, vehicles, roadway environment and road users) and contributory factors that could have lead to the crash. The contributory factors are recorded according to the SafetyNet Accident Causation System (SNACS). The development of this system will be described in the next section.

\section{SafetyNet Accident Causation System (SNACS)}

The accident model underlying SNACS is based on a contemporary accident model [1], framed in a Man-Technology-Organisation (MTO) perspective. When applied to the road-research domain, their equivalents are:

M (Man): the driver's cognitive functions; observation, interpretation, planning and actions. Temporary and permanent driver states.

$\mathrm{T}$ (Technology): the vehicle's equipment and interface, physical driver environment

O (Organisation): road environment, ambient conditions, communication, driver training.

Drivers' erroneous actions are viewed as indicators of mismatches between driver, technology and environment, rather than as errors per se. Therefore the philosophy behind SNACS is that road traffic crashes are the result of a failure in the dynamic process of interaction between humans, technology and organisation (incl. 
environment). This approach has been in use for some time in domains where unwanted system states can yield negative consequences on a large scale, such as nuclear power plants and aviation, but is relatively new to the field of road safety.

This is reflected in the origins of the SNACS method. The Cognitive Reliability and Error Analysis Method (CREAM) uses the MTO perspective to analyse the contributory factors leading to industrial accidents such as those occurring in nuclear power plants [1]. CREAM was adapted to be applicable for the analysis of road traffic crashes and became DREAM (Driver Reliability and Error Analysis Method) [3]. DREAM was translated into English and adapted for use within the SafetyNet project, taking on the name SNACS. (For further details see [4]).

The accident model underlying SNACS distinguishes between sharp end and blunt end failures. A crash is caused by a failure in the interactions between Man, Technology and Organisation at a specific point in time and space (sharp end). However the model extends beyond this to consider the contributory factors that may be more remote in time and space but the consequences of which can form negative settings that facilitate accident occurrence ([5], [1], [6])). The accident model is illustrated in Figure 1.

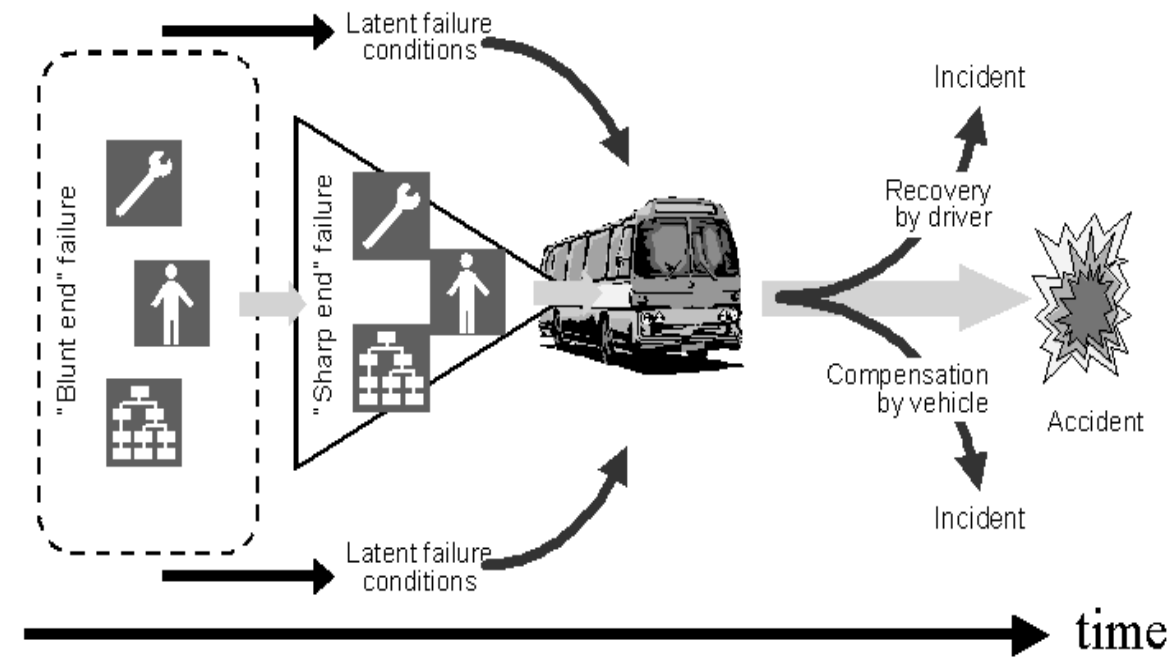

Figure 1 Accident model underlying SNACS - Adapted from [6]

The advantage of this approach is that it regards the driver's actions in a larger context than is often used, showing how a quite large scope of factors can contribute to the occurrence of crash. It also points out that problems arise as a consequence of the interactions between driver, vehicle and road environment, and that multiple factors can contribute to the same crash. SNACS is a tool used to classify these factors and allows links between them to be identified.

The coding system distinguishes between critical events (sharp end failures) and causes (blunt end failures). A critical event is the observable consequence that leads to the crash and is expressed in terms of time, space or energy. Causes are the contributing factors that lead to this event. Causes are organised into groups and represent organisation, infrastructure, vehicle or road user related factors. Examples 
of the critical events and causes are shown in Table 1. SNACS analysis is performed on a vehicle level, based on the assumption that each active road user can contribute to a crash. Therefore SNACS charts are recorded for each driver, rider and pedestrian - that is the road users with the primary control over the vehicle they are operating. SNACS is performed when as much data as possible about the crash has been collected and the investigator uses all data to inform the SNACS coding.

Table 1 Examples of critical event and cause codes

\begin{tabular}{|c|c|c|c|c|}
\hline \multicolumn{4}{|c|}{ Causes (contributory factors) } & \multirow{2}{*}{$\begin{array}{c}\text { Critical } \\
\text { Events } \\
\begin{array}{c}\text { Observable } \\
\text { Effects }\end{array}\end{array}$} \\
\hline Organisation & Infrastructure & $\begin{array}{c}\text { Vehicle (Incl } \\
\text { HMI) }\end{array}$ & Road User & \\
\hline $\begin{array}{l}\text { M1 Deficient } \\
\text { instructions/ } \\
\text { procedures } \\
\text { M4 } \\
\text { Inadequate } \\
\text { training }\end{array}$ & $\begin{array}{l}\text { J2 } \\
\text { Communication } \\
\text { failure (driver- } \\
\text { environment) } \\
\text { K2 Maintenance } \\
\text { failure - } \\
\text { condition of road } \\
\text { N2/4Obstruction } \\
\text { of view } \\
\text { N5 Inadequate } \\
\text { roadside design }\end{array}$ & $\begin{array}{l}\text { G1 Access } \\
\text { limitations } \\
\text { H2 Illumination } \\
\text { I1 Equipment } \\
\text { failure } \\
\text { K1 Maintenance } \\
\text { failure - } \\
\text { condition of } \\
\text { vehicle } \\
\text { O2 Inadequate } \\
\text { HMI }\end{array}$ & $\begin{array}{l}\text { B1 Observation } \\
\text { missed } \\
\text { C1 Faulty } \\
\text { diagnosis } \\
\text { D1 Inadequate } \\
\text { planning } \\
\text { E3 Distraction } \\
\text { E6 Inattention } \\
\text { F1 Functional } \\
\text { Impairment } \\
\text { J1 } \\
\text { Communication } \\
\text { failure (driver- } \\
\text { driver) } \\
\text { L2 Insufficient } \\
\text { knowledge }\end{array}$ & $\begin{array}{l}\text { A1 Timing } \\
\text { A2 Duration } \\
\text { A3 Force } \\
\text { A4 Distance } \\
\text { A5 Speed } \\
\text { A6 Direction } \\
\text { A7 Object } \\
\text { A8 Sequence }\end{array}$ \\
\hline
\end{tabular}

The SNACS analyst works backwards in time from the point at which the vehicle loses control and a crash is inevitable. First, the driver/rider/pedestrian is assigned one critical event. Then one or several causes (Cause Z), to which the critical event is a consequence of, are added. Then any causes that contributed to the cause $Z$ are added and so on. This process produces a causation chart (see Figure 2) where $Y$ is a consequence of $X$ and a cause to $Z$. Coding conventions guide when the chart is complete, which causes can directly lead to a critical event, and which causes can directly lead to a particular consequence. Subcategories of critical events and causes (indicated with dashed lines in Figure 2) are assigned to give more detail. When a subcategory is added that part of the chart is stopped. For more information on SNACS please see the SNACS manual within the SafetyNet Deliverable 5.5 [7]. 


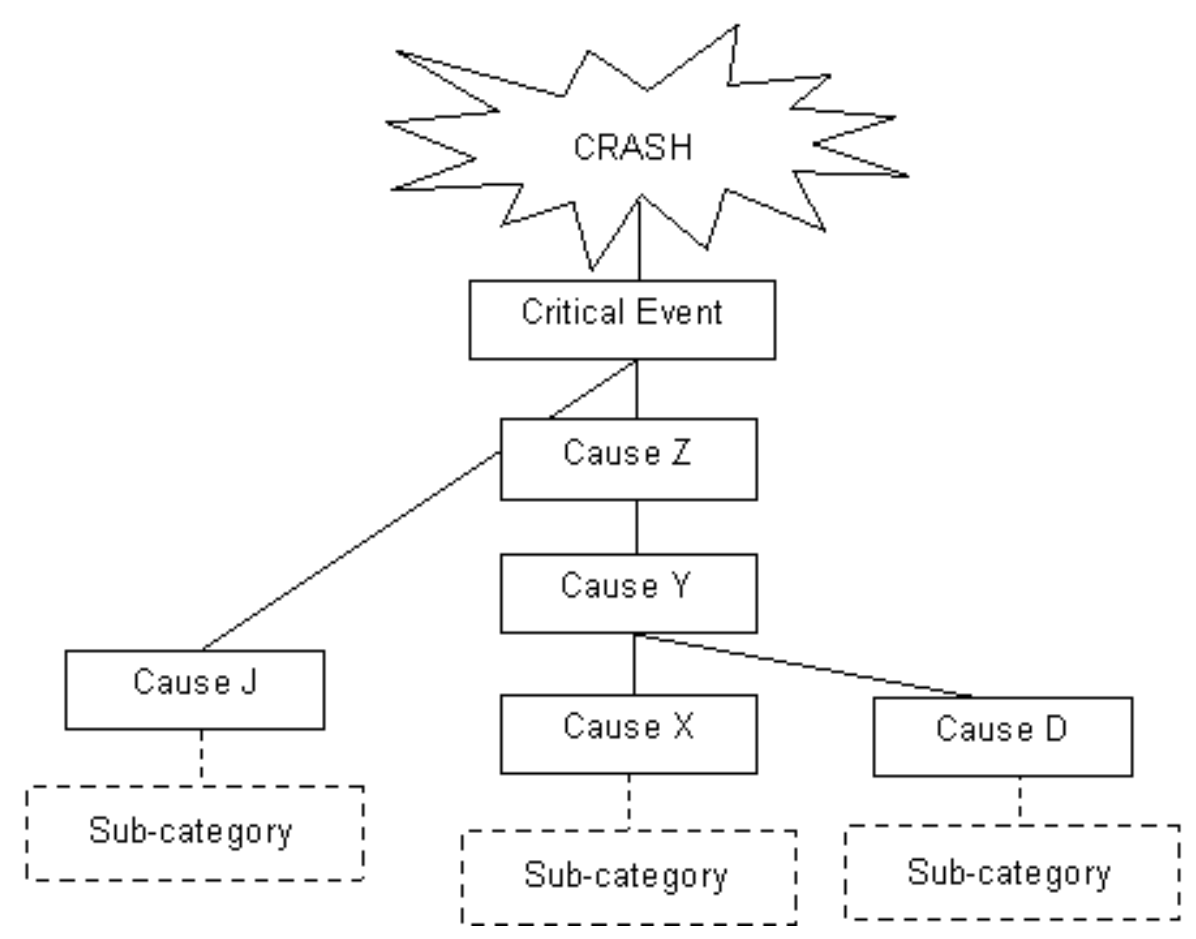

Figure 2 Example SNACS chart for one driver/rider/pedestrian

The analysis of aggregated cases is performed by superimposing individual charts, in order to find common causation patterns for a selected group of cases. Comparisons can be made between the diagrams of different groups of drivers/riders/pedestrians.

\section{Analysis of Distraction and Inattention in the Accident Causation Database}

The contributory factors 'E3 Distraction' and 'E6 Inattention' were both included in the database as 'temporary personal factors'. Distraction was defined as when

'the performance of a task is suspended because the person's attention was caught by something else or the attention has shifted'

Aspects that could distract include conversations with passengers, objects outside of the vehicle, answering a mobile phone and the road user thinking about something that is unrelated to the driving task.

Inattention is defined as 'Low vigilance due to loss of focus'. Aspects that could lead to inattention include coughing; driving on a boring road, i.e. where the road features and environment remain the same for an extended period; and over familiarity with the journey, e.g. not noticing a sign had changed.

Cases have been selected for the following analysis when a driver, rider or pedestrian has been assigned the codes 'Distraction' or 'Inattention'. Collision partners which were not assigned these codes were excluded. 


\section{RESULTS}

Out of the 1005 crashes recorded in the SafetyNet Accident Causation Database, $320(32 \%)$ involved at least one driver, rider or pedestrian which had been assigned the SNACS codes 'Distraction' and/or 'Inattention'. These crashes involved 633 vehicles and pedestrians. 212 of the drivers/riders/pedestrians were assigned the code 'Distraction', 140 were assigned 'Inattention'. As only six drivers/riders/pedestrians were included in both the 'Distraction' and 'Inattention' group, it can be said that these contributory factors make up two relatively distinct groups within the databases. The six drivers/riders/pedestrians which were assigned charts that include both the causes 'Distraction' and 'Inattention' have been included in both the Distraction and Inattention groups in the following analysis.

Figure 3 shows the percentage share of each vehicle type within the 'Distraction' and 'Inattention' groups. The Car/MPV group have the largest share for both vehicle types and this reflects the distribution of vehicle types in the database as a whole. There are small differences in the share of other vehicle types. Motorcycles/mopeds form a greater share of the 'Inattention' group than the 'Distraction' group and a greater percentage of pedestrians were assigned the code 'Distraction' than were assigned 'Inattention'.

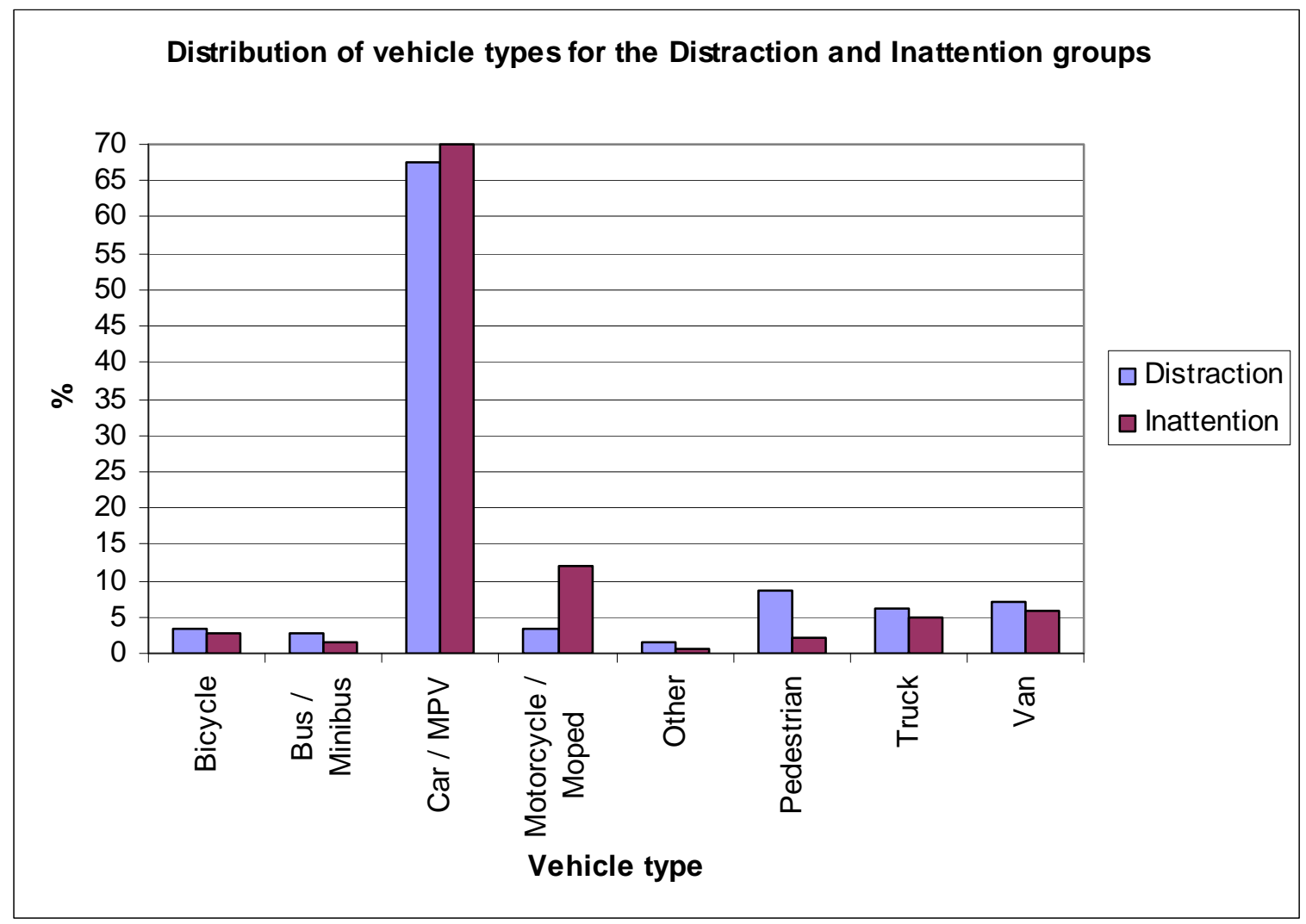

Figure 3 The distribution of vehicle types for vehicles assigned the SNACS code Distraction and Inattention groups 


\section{Contributory factor patterns - SNACS Diagrams}

The SNACS charts for drivers, riders and pedestrians with the SNACS code 'Distraction' are shown in Figure 4 and Figure 5 and for 'Inattention' in Figure 6 and Figure 7. These diagrams are created by aggregating or 'overlaying' the SNACS charts for each individual driver/rider/pedestrian (see Figure 2). The thickness of the lines linking each cause/critical event reflects the number of drivers/riders/pedestrians who had that particular link in their individual SNACS chart. Figure 4 and Figure 6 show the critical event to cause links with the number of drivers, riders and pedestrians that have been assigned each critical event in brackets. Several causes can contribute to a critical event.

Figure 5 and Figure 7 show the cause to consequence links. In these figures, CEL has been used to indicate the causes which have links with critical events and the number of these links. For all figures the number in the boxes shows the number of links. Any link that only occurred once or twice is shaded in grey.

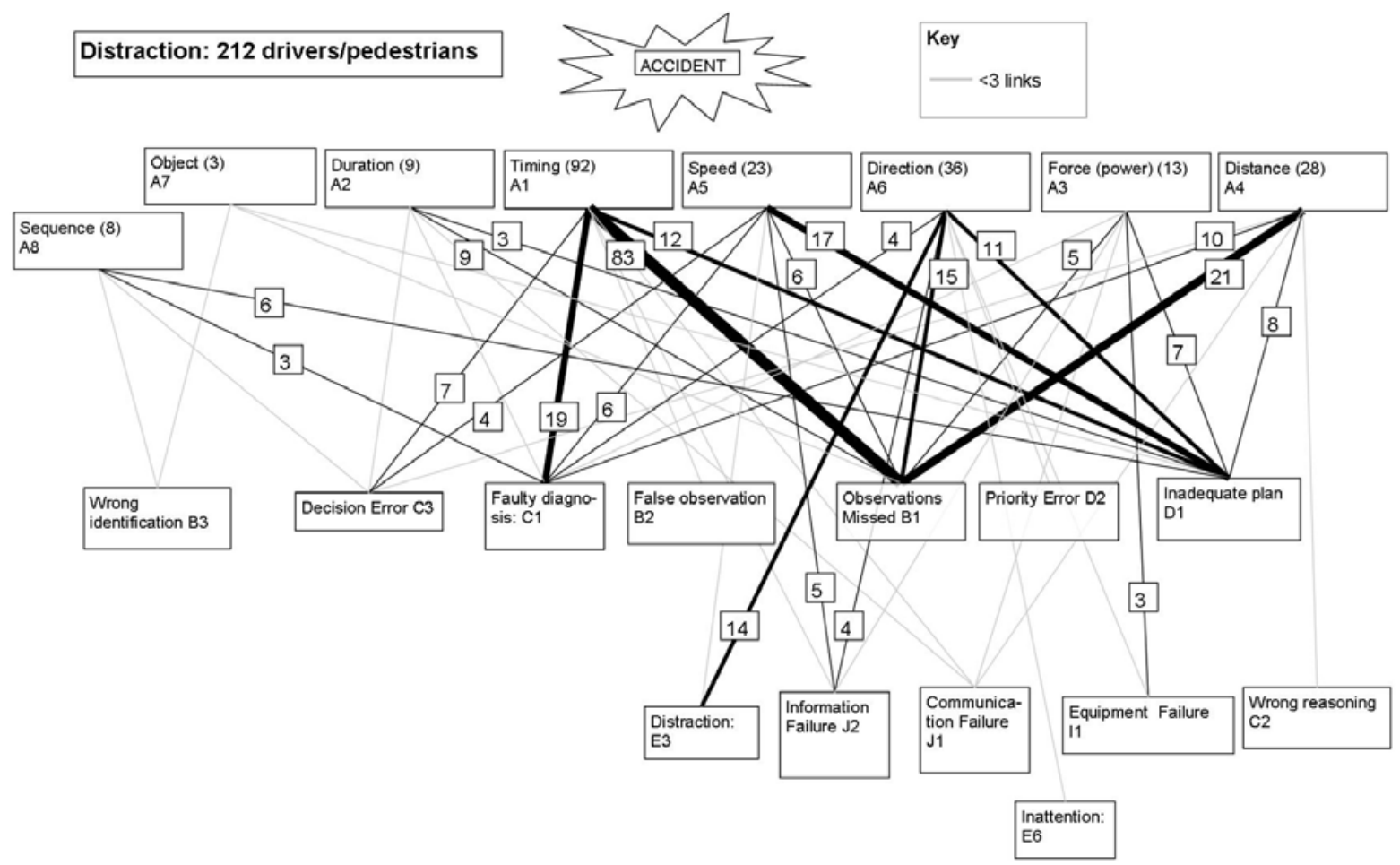

Figure 4 Critical event to cause links for 'Distraction' drivers/riders/pedestrians (factors leading directly to the crash)

Figure 4 shows that the strongest pattern is 'Observation missed' to 'Timing' with this link being coded in 39\% of the 'Distraction' drivers/riders/pedestrians. An example is when a driver misses a junction sign (Observation missed) and brakes too late (Timing) to avoid moving into the path of another vehicle. 'Observation missed' to 'Distance' is also a strong pattern. 'Distraction' is only linked directly with the critical events 'Direction' and 'Speed' although the latter is a very weak pattern. Therefore in this sample the most commonly occurring sharp end failure was 'Timing' e.g. 
applying the brakes too late with the blunt end failure 'missed observation' being a direct contributor.

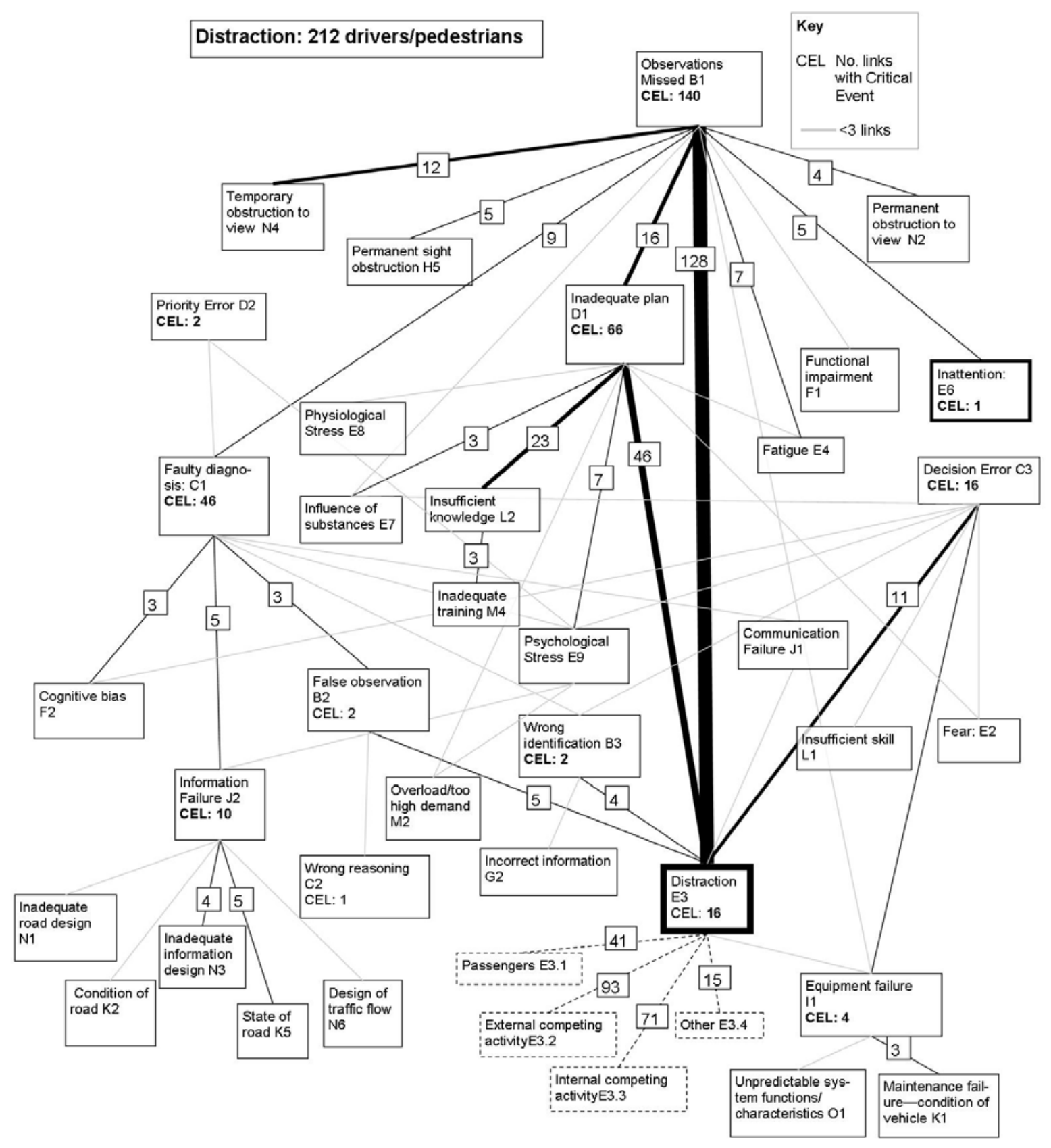

Figure 5 Cause to Cause pattern for 'Distraction' drivers/riders/pedestrians (factors contributing to the crash)

Figure 5 demonstrates that when considering the wider contributors to a crash (blunt end failures) there are many different factors that can lead to a crash. The strongest patterns for 'Distraction' are the links with 'Observation missed' and 'Inadequate plan'. The diagram shows that in the sample of drivers/riders/pedestrians, 'Distraction' was a cause of 'Observation missed' 128 times and 'Inadequate plan' 46 times. In turn 'Observation missed' was a consequence of 'Inadequate plan' 16 
times. Other factors that contribute to missed observations include temporary or permanent sight/view obstructions and 'Inadequate plan'. 'Insufficient knowledge' is a contributing factor that also has a fairly strong influence in this causation pattern. This means that 'Distraction' was an important direct cause of missed observations but that other factors such as sight obstructions also contribute to missed observations in for some drivers/riders/pedestrians. The influence of 'Insufficient knowledge' is important as this indicates that for example being unfamiliar with the road is associated with accidents involving distraction. Figure 5 also shows the types of distraction that were recorded in the sample. These are indicated by dashed lines and 'External competing activity' was the most common type of distraction. By joining figure 3 and figure 4 together, the strongest pattern is that drivers/riders/pedestrians are distracted because of an 'External competing activity' and miss an observation which leads to a 'Timing' (premature, late or no action).

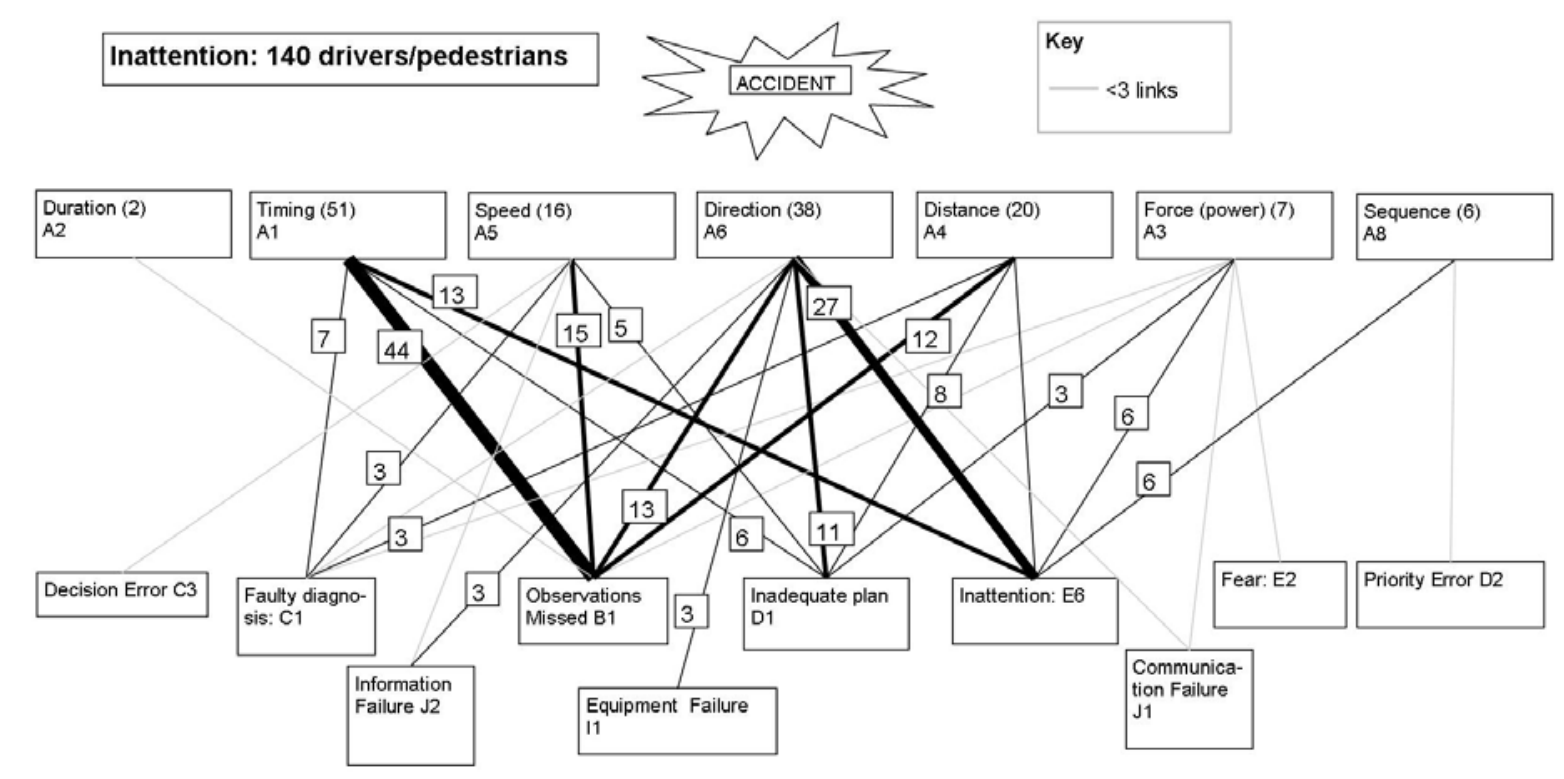

Figure 6 Cause to critical event links for 'Inattention' drivers/riders/pedestrians (factors leading directly to the crash)

Figure 6 shows that the strongest cause to critical event pattern recorded for drivers, riders and pedestrians with the SNACS code 'Inattention' are 'Observations missed' to 'Timing' (44 times) and 'Inattention' to 'Direction' (27 times). So similarly to distraction crashes, 'Timing' is a common sharp end failure for inattention crashes. To fully make sense of this diagram, it has to be considered along side Figure 7 . 


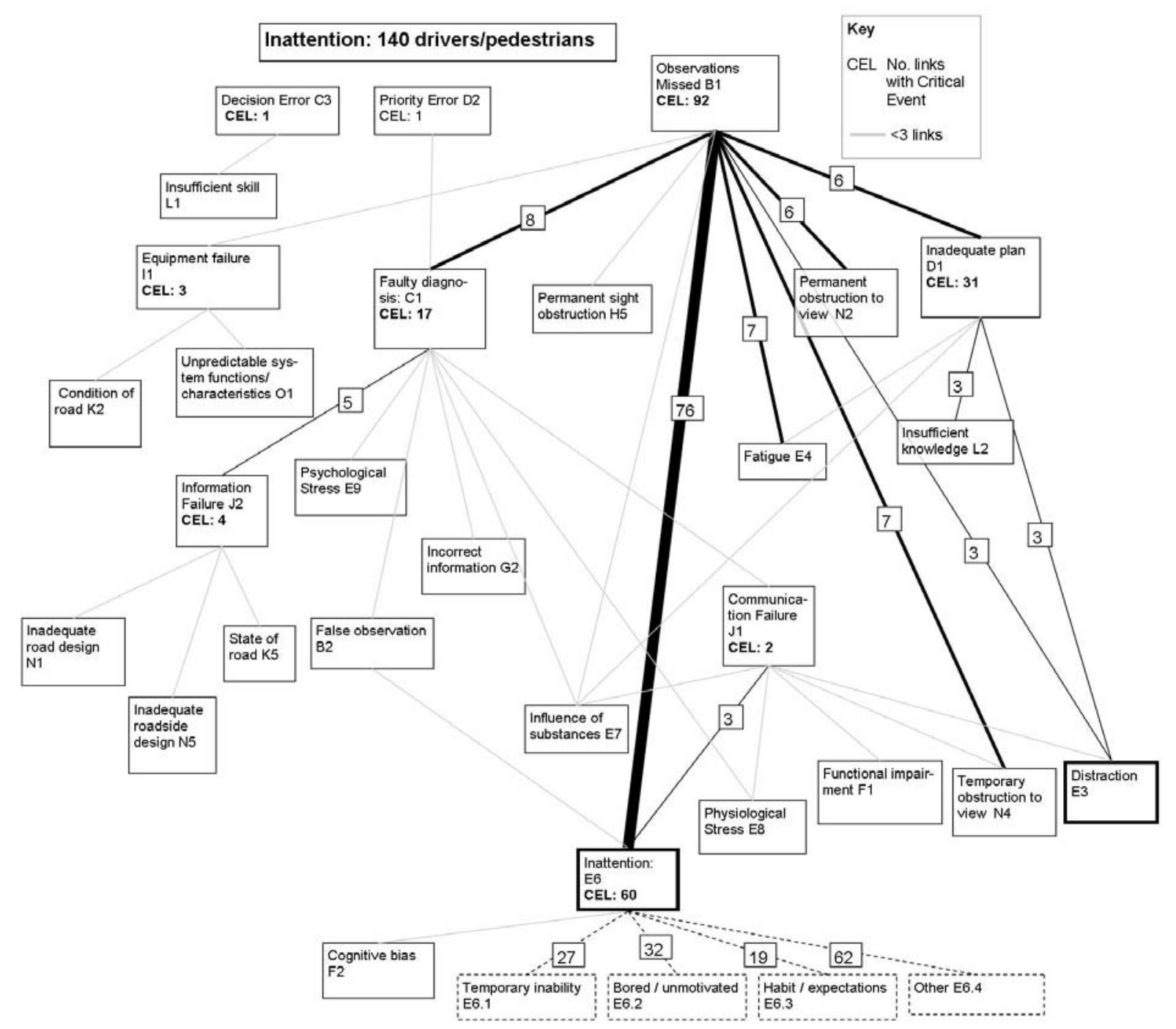

Figure 7 Cause to cause links for 'Inattention' drivers/riders/pedestrians (factors contributing to the crash)

Figure 7 highlights that 'Inattention' was observed to contribute to 'Observation missed' many times. Again similarly to the 'Distraction' group the SNACS codes, temporary and permanent sight/view obstruction factors are linked to 'Observation missed'. When examining both Figure 6 and Figure 7, it appears that 'Inattention' to 'Observation missed' to 'Timing' is the most common pattern however 'Inattention' links directly to the critical events (60 links) nearly as many times as it does to 'Observation missed' (76 links). So for inattention accidents sharp end failures can be as a direct or indirect consequence of 'Inattention'. Direct comparisons between the 'Inattention' to critical event links and 'Distraction' to critical event links cannot be made as SNACS coding conventions do not allow direct linking between the cause 'Distraction' and critical events. This is a potential limitation of the method which has been addressed by updating SNACS. This update will be discussed further in the Discussion and Conclusions section.

An individual SNACS chart ends with cause subcategories that give more detail about the causes, which start the chain of events that leads to the crash. As can be 
seen in Figure 5 and Figure 7, in the majority of cases 'Distraction' and 'Inattention' respectively are positioned in the chart at the furthest position from the critical events, i.e. these causes are rarely a consequence of another cause. Additional information about what caused the 'Distraction' and 'Inattention' in the two groups can be gained by examining the 'Distraction' and 'Inattention' subcategories, as displayed in Figure 5 and Figure 7 and indicated with dashed lines.

More than one subcategory can be coded for each driver/rider/pedestrian and 'Other' is coded either when the investigator is not sure what distracted or lead to inattention or none of the subcategories accurately described it. Figure 5 shows that for distraction, 'External competing activity' is the most frequently coded subcategory followed by 'Internal competing activity'. These activities can be related or unrelated to the driving tasks. External activities could range from trying to read road signs to the driver/rider/pedestrian recognising someone they know. Internal activities include using a mobile phone, operating a satellite navigation system or becoming lost in thought, but conversations with other people in the vehicle would be coded 'Passengers'. This sample suggests that what occurs within a vehicle is more likely to distract a driver/rider than external factors.

For Inattention Figure 7 shows that 'Other' is coded most frequently. 'Bored' is the next most frequent subcategory followed by 'Temporary inability' (e.g. coughing, sneezing). A driver/rider/pedestrian may receive a 'Bored' code if the journey they have taken is very familiar e.g. journey to work, which may cause them to lose concentration on what is going on in the roadway environment. This overlaps with the 'Habit' category as 'Habit' is when a road is familiar so the driver/rider/pedestrian expects everything to remain the same so may miss changes to road signs or priorities etc.

\section{Crash type in relation to Distraction and Inattention}

It has been demonstrated in previous analyses of the SafetyNet Accident Causation Database that SNACS chart patterns differ according to the context of the crash and the trajectory of the involved vehicles [8]. Therefore to identify any differences between the Distraction and Inattention groups, each driver, rider and pedestrian were assigned to one of the four categories that were included in previous analysis. Three groups were related to vehicle trajectory. Each relevant vehicle was assigned to one of these groups individually so in some cases, two vehicles that were involved in the same collision were assigned to different trajectory categories. The forth group included all pedestrians, cyclists and their collision partners under the heading 'Vulnerable road users'. These 'slower moving' vulnerable road users were treated separately because of the belief that crashes involving this category of road user would have different causation patterns and characteristics when compared to single or multiple motorised vehicle crashes. The 4 categories are as follows:-

- Crossing paths: when the driver performs a manoeuvre that potentially crosses the path of another road user and has a crash as a result (e.g. intersection crashes)

- Leaving lane: when the vehicle either moves into another lane, crosses the median line or runs off the road and as a result has a crash 
- Own lane: when a driver is in collision with an object/vehicle in its own lane and has not previously left its lane.

- Vulnerable Road Users: Pedestrians, Pedal cyclists and their collision partners (in this case motorcycles were included in the trajectory groups)

The distribution of these categories within the Distraction and Inattention groups are shown in Figure 8.

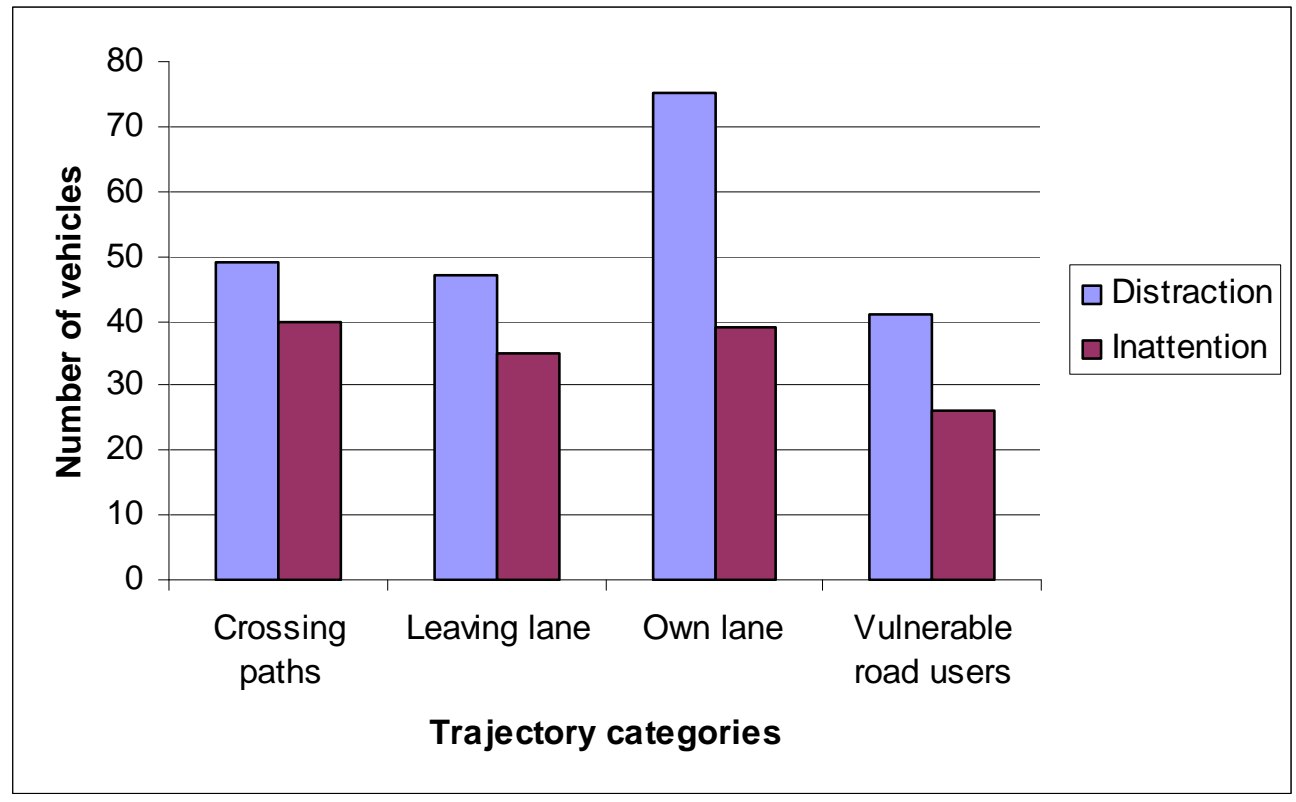

Figure 8 Inattention and Distraction groups by trajectory

Figure 8 shows that the 'Inattention' vehicles are distributed fairly evenly between the trajectory categories. However, for the 'Distraction' vehicles the category 'Own lane' includes more vehicles than any of the other crash types. Figure 9 shows how the 'Distraction' and 'Inattention' subcategories are distributed within each crash type. The $y$-axis indicates percentage and the numbers within the bars represent the number of vehicles and pedestrians. 


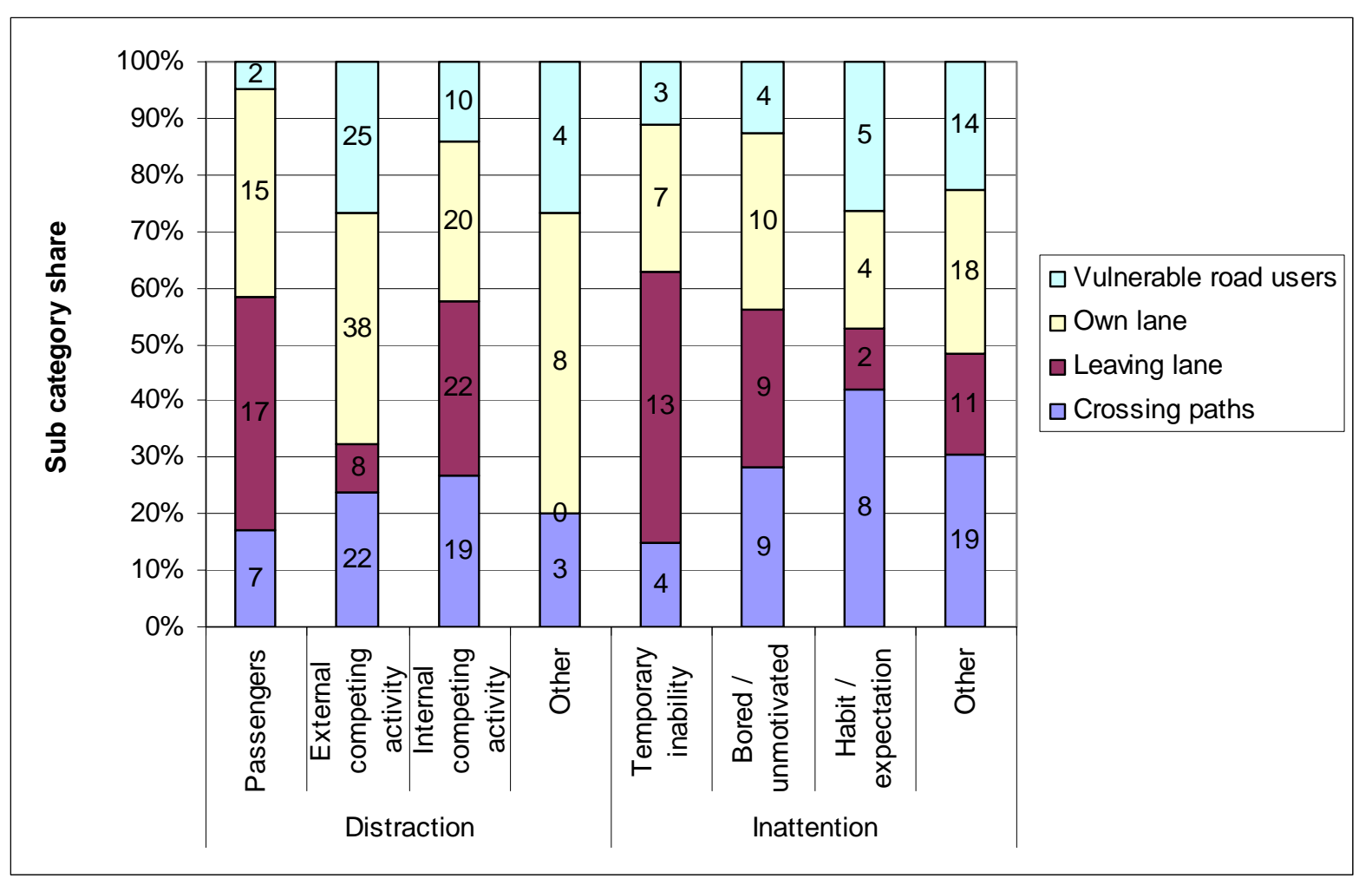

Figure 9 Distraction and Inattention subcategories by crash type

Figure 9 indicates that within the 'Distraction' group, a slightly larger share of the 'Internal competing activity' and the 'Passenger' subcategories are assigned to 'Leaving lane' vehicles as opposed to vehicles with other trajectories. In contrast the subcategory 'External competing activity' was more frequently assigned to 'Own lane' vehicles. The 'Vulnerable road users' category has a larger share of the 'External competing activities' than the 'passengers' and 'Internal competing activity' subcategories.

The figures for the 'Inattention' group shown in Figure 9 are more difficult to asses due to the number of times 'Other' was assigned. 'Temporary inability' appears to more frequently associate with 'Leaving lane' vehicles than the other categories. 'Habit / expectation' has the largest share of both the 'Crossing paths' and 'Vulnerable road users' categories.

\section{DISCUSSION AND CONCLUSIONS}

212 drivers/riders/pedestrians had 'Distraction' included in their SNACS charts and 140 had 'Inattention' as a cause in their SNACS charts, making 'Distraction' more prevalent than 'Inattention' in the SafetyNet Accident Causation Database. The distribution of vehicle type is similar across both groups. However, there is a slightly higher percentage of Motorcycles/mopeds in the 'Inattention' group and pedestrians in the 'Distraction' group. This may relate to the SNACS definitions where 'Distraction' relates to attention being caught by an external object or internal thought whereas 'Inattention' relates to a lack of concentration rather than a specific 
distraction. It may be that pedestrians are more prone to environmental distractions and preoccupations rather than a lack of concentration. Motorcycle/moped riders on the other hand may be less likely to be distracted by external factors - for example it is more difficult to converse with a pillion passenger than a car/van passenger. However further conclusions cannot be drawn due to the small numbers observed.

The SNACS diagrams show that both 'Distraction' and 'Inattention' most frequently lead to missed observations of some kind which in turn lead to a critical event of 'Timing' (premature, late or no action) or (incorrect) 'Direction'. They also show that there are many other contributory factors, which interact or co-exist with these 'causes'. This is particularly apparent with the 'Distraction' group; however this is likely to be because there are more vehicles and therefore more individual SNACS charts included in the 'Distraction' chart when aggregated. Factors such as permanent and temporary obstructions to view co-exist with both 'Distraction' and 'Inattention' and it is easy to see that these factors could exacerbate the consequences of becoming distracted or loosing concentration. 'Insufficient knowledge', which refers to the driver/rider/pedestrian being unfamiliar with the roads on which they are travelling, also showed a fairly strong pattern for vehicles whose accidents involved 'Distraction'. The demands of driving become greater when the environment is unfamiliar. For example the driver does not know the layout of the road ahead and must read signs to navigate successfully. These factors make it more likely for the driver to become distracted from the immediate driving task and would make the impact of additional factors such as talking to a passenger or on a mobile phone greater.

With one exception, 'Distraction' and 'Inattention' drivers/riders/pedestrians are distributed fairly equally between the crash type categories (Crossing paths, Leaving lane, vulnerable road users - excluding powered 2 wheelers). The exception was the own lane category, which had a greater number of the 'Distraction' drivers/riders/pedestrians than the other categories. There were also associations between the crash types and specific distraction or inattention subcategories. This suggests that countermeasures to these subcategories may lead to bigger reductions of crashes with certain characteristics than others. For example leaving lane crashes in the database have been associated with young males [8]. When looking at the distraction subcategories 'passengers' and 'internal competing activates', these show a greater association (albeit slight) with leaving lane crashes than other accident types. 20 out of the 39 drivers of vehicles in this category are aged 25 or less, of which $70 \%$ are male. It is possible that the young male group are more likely to be distracted by passengers or more likely to use mobile phones or navigation aids whilst driving although generalisations are difficult with such small numbers.

The analysis reported here both gives an idea of the prevalence of the contributory factors 'Distraction' and 'Inattention' in the SafetyNet Accident Causation Database and demonstrates the type of information available through SNACS analyses. As with any dataset however, it does have limitations. Generalising from the data has to be done with care. The data are not necessarily truly representative of the countries where they were collected. This is due in part to complexity and expense of the collection methodologies. The database is also not representative of Europe as only 6 of the member states are included and these are biased towards the best performing countries in terms of road safety. There was, however, good intercoder 
reliability meaning that the reliability of the SNACS data is high [9]. An evaluation of the SNACS codes was also undertaken to assess the likely validity. This was achieved by comparing the SNACS codes and definitions to evidence of accident causation and contributory factors found in relevant literature [10]. This formed part of a wider assessment of the SNACS method, which lead to a refinement of the method to become DREAM 3.0 [11]. Changes included the removal of codes, which had very little supportive evidence in the literature, altering of definitions to be more meaningful and the merging of codes which were found to overlap both by the SNACS coders and the literature.

These refinements lead to a merging of the 'Distraction' and 'Attention' codes in a single code 'Inattention'. The five subcategories for 'Inattention' in DREAM 3.0 distinguish between driving and non-driving related factors and whether these occurred inside or outside of the vehicle as well as having a distinct subcategory 'Thoughts/Daydreaming'. This removes some of the potential confusion in SNACS caused by subcategories such as 'internal competing activity', which for example could either mean distraction due to using a navigational device or getting lost in thought.

In conclusion, the SNACS method, continued as DREAM 3.0, has great potential to give detailed information about how distraction/inattention or other factors contribute to road traffic crashes. The data can be used to identify which road user groups are most likely to be involved in which crash type due to distraction/inattention and give detailed information to assist in the development of countermeasures. As the method aims to capture all the contributory factors involved in the crash it gives information about how these may interact. When included in a large dataset, the SNACS charts give an idea of the order in which events happen in the lead up to a crash which provides valuable information about which point in time the application of a countermeasure, especially primary safety countermeasures, would be most affective.

\section{ACKNOWLEDGEMENTS}

The authors would like to acknowledge the contribution of the SafetyNet Work Package 5.2 partners to the development of the SafetyNet Accident Causation Database, the SafetyNet Accident Causation System and SNACS analysis methods:

K. Björkman, M. Ljung Aust, E. Liljegren, G. Björklund (Chalmers); A. Morris, R. Danton (VSRC); G. Giustiniani, D. Shingo Usami (DITS); K. Parkkari (VALT); M. Jaensch (MUH); E. Verschragen (TNO). Additional thanks go to Mikael Ljung Aust for his contributions to this paper. 


\section{REFERENCES}

[1] Hollnagel, E. Anticipating failures: What should predictions be about? In Proceedings of the Human Factors and Medicine Panel (HFM) Workshop, (Siena, 1999).HFM

[2] Hollnagel, E, Cognitive Reliability and Error Analysis Method: CREAM. Elsevier Science Ltd, Oxford, UK, 1998

[3] Ljung, M, DREAM: Driving Reliability and Error Analysis Method. (Master's thesis), Linkoping University, Linkoping, 2002

[4] Fagerlind, H, Bjorkman, K, Wallén Warner, H, Ljung Aust, M and Sandin, J, Morris, A, Talbot, R, Danton, R, Giustiniani, G, Shingo Usami, D, Parkkari, K, Jaensch, M, Verschragen, E, Development of an In-depth European Accident Causation Database and the Driving Reliability and Error Analysis Method, DREAM 3.0. in Proceedings of Expert Symposium on Accident Research (ESAR) $3^{\text {rd }}$ International Conference (Hannover, 2008) BASt.

[5] Reason, J. Human Error. Cambridge University Press, Cambridge, 1990

[6] Hollnagel, E. Barriers and Accident Prevention. Ashgate Publishing Ltd, Aldershot, 2004

[7] SafetyNet, Glossary of Data Variables for Fatal and accident causation databases. Deliverable 5.5 of the EC FP6 project SafetyNet*, 2008

[8] SafetyNet, In-depth accident causation database and analysis report. Deliverable D5.8 of the EC FP6 project SafetyNet* ${ }^{\star} 2008$

[9] Wallén Warner, H, Sandin, J, The intercoder agreement when using the Driving Reliability and Error Analysis Method in road traffic accident investigations, Paper submitted for publication

[10] Wallén Warner, H, Björklund, G, Johansson, E, Ljung Aust, M, Sandin, J, DREAM 3.0; Documentation of references supporting the links in the classification scheme, 2008

[11] H Wallén Warner, G Björklund, E Johansson, M Ljung Aust, J Sandin, Manual for DREAM 3.0 Driving Reliability and Error Analysis Method. Deliverable D5.6 of the EC FP6 project SafetyNet*, 2008

*All SafetyNet deliverables are available at www.erso.eu 